According to $\mathrm{C} \& \mathrm{~W}$, sentence (1) is made up of just one proposition, but sentence (2) is made up of two. The idea seems to be that in sentence (1) the boy is simultaneously hugging the girl and the baby (the three are squeezed into one bear hug), but in sentence (2) the boy hugs a girl and also kisses a baby (but notice he could do both at the same time; he could be hugging both, but kissing only the baby). The claim that there is only one proposition in sentence (1) assumes that comprehenders create a particular mental model for the sentence, one in which a boy hugs both a girl and a baby at the same time. The meaning of the sentence is also compatible, though, with a situation in which the boy hugs the girl and then he hugs the baby, in which case clearly the sentence contains two propositions. It could even be argued that the sentence contains two propositions even in the simultaneous case, because the representation indicating the meaning of the sentence has to indicate somewhere what agent acted on the baby. The point here is not that the analysis assumed by $\mathrm{C} \& \mathrm{~W}$ is wrong but rather that their position requires a set of assumptions that are vulnerable to challenge and so must be explained in more detail.

I will end by repeating that I generally like the approach the authors take in this work. Recently we have begun examining how unimpaired undergraduates interpret unambiguous sentences such as The lawyer was sued by the doctor and The dentist was pulled by the tooth. We are finding striking evidence that sentences that are implausible by virtue of world knowledge - in the authors' terminology, by post-interpretive processing - are processed quite differently from sentences whose sensibility can be ruled out based on feature mismatch (a tooth cannot be an agent because agency requires animacy). Even more intriguing is our finding that, if the two participants in an implausible event (e.g., The lawyers were sued by the doctor) differ on some grammatical feature (plurality in this case), confusion occurs much less often than when the noun phrases cannot be distinguished with a grammatical feature. We believe the distinguishing features allow the two noun phrases to be unambiguously bound to their appropriate thematic roles, and as a result misinterpretations are much less likely to occur (the two noun phrases cannot swap positions). Data of this sort provide independent and compelling evidence for the original and creative theoretical perspective $C \& W$ present in their target article, because they require an explanation that assumes a separation between processes that compute interpretations and so are internal to the language system, and processes that evaluate and modify those interpretations and are therefore external to the system.

\section{Modularity, segregation, and interactions}

\section{Karl J. Friston \\ The Wellcome Department of Cognitive Neurology, Institute of Neurology, London WC1N 3BG, England. k.friston@fil.ion.ucl.ac.uk}

\begin{abstract}
This commentary considers how far one can go in making inferences about functional modularity or segregation, based on the sorts of analyses used by Caplan \& Waters in relation to the underlying neuronal infrastructure. Specifically, an attempt is made to relate the "functionalist" approach adopted in the target article to "neuroreductionist" perspectives on the same issue.
\end{abstract}

Caplan \& Waters $(\mathrm{C} \& W)$ provide compelling arguments in support of the notion that there is a separate working memory system for assigning the syntactic structure of a sentence by noting that there is no interaction between structural complexity and other processes that require working memory. This is a nice example of a general approach to inferring something about the cognitive architecture of a system given only its outputs (responses) to inputs or stimuli that can be manipulated over a number of dimensions. The approach is related to Sternberg's revision of Donder's subtractive method (Sternberg 1969) in which the interactions among various processes are used to infer something about their functional organization. Both depend critically on interactions, and it is this theme that will be pursued in the context of neurobiologically motivated analyses of functional anatomy. The key question, addressed by $\mathrm{C} \& \mathrm{~W}$, is whether the mnemonic aspects of interpretive sentence processing are modular or functionally dissociable from other (e.g., post-interpretive) aspects. This question is purely functionalist but implies the existence of distinct neuronal systems that mediate this processing. An explicit version of the same question is "Is there functional segregation, in terms of neuronal systems, for the structural complexity of sentences?" Much is invested in the term "functional segregation" here, and it is worthwhile considering what it means.

The brain appears to adhere to two principles of organization, functional segregation (Phillips et al. 1984) and the functional integration of segregated systems, such as cortical areas, subareas, neuronal populations or individual cells. "Functional segregation" refers to the selective neuronal responses to specific sensorimotor attributes. Consider the cortical area V5, a motion-sensitive area (Zeki et al. 1991) that can be characterized in terms of population responses (as measured with fMRI or local field potentials with electrode recordings) to visual stimuli. To demonstrate functional specialization for motion, one would have to show a high degree of mutual information between V5 responses and visual motion. For this area to be functionally segregated, there is a further requirement that there are no responses to other attributes, such as changes in colour. This constraint has important consequences for the context sensitivity of speed-dependent responses: imagine that we characterised the receptive field $f(\mathrm{~s}, \lambda)$ of a V 5 cell as a function of stimulus speed (s) and wavelength $(\lambda)$. Under segregation, the responses (x) over different speeds should be the same for any two wavelengths:

$$
\mathrm{x}=f\left(\mathrm{~s}, \lambda_{1}\right)=f\left(\mathrm{~s}, \lambda_{2}\right)=f(\mathrm{~s}) .
$$

In short, the speed-dependent responses would be insensitive to the colour of the stimulus. In other words, functional segregation for a specific attribute implies that the same responses will be elicited by changes in that attribute irrespective of the other stimulus attributes (or more generally the context). Imagine that we measured the response of $\mathrm{V} 5$ to a changes in speed using long-wavelength (red) stimuli and short-wavelength (blue) stimuli. If we found a response difference (i.e., a context-sensitive, speed-dependent response), then functional segregation for motion per se has to be rejected and we would infer that this area preferred red (or blue) motion (see Fig. 1 for a schematic illustration of the receptive fields implied by these situations). This context sensitivity is simply revealed by the interaction between speed and wavelength in predicting the response. Precisely the same construct is used by C\&W. In their formulation the response is in terms of speed and accuracy of sentence processing and the stimulus attribute of interest is structural complexity. They have examined the complexity-dependent responses (low vs. high) in two contexts to see if there was an interaction. Failing to find an interaction allows them to assert that functional segregation is a sufficient model for the observed findings. The two contexts were low and high concurrent working memory load or subjects with low and high capacity.

The above argument suggests that functional segregation should involve a series of simple subtractions in different contexts in order to demonstrate the context-invariant nature of selective responses. In functional neuroimaging this is the tenet of "conjunction analyses" (Price \& Friston 1997). A conjunction is defined as the presence of a main effect (e.g., of structural complexity) in the absence of interactions (e.g., with memory load). It would be interesting to revisit this issue with functional neuroimaging using a factorial design wherein structural complexity (high and low) was crossed with memory load (sub- and supraspan). It is possible that some brain regions would show functional segregation for complexity (as identified with a conjunction analysis). However, it is likely that some regions would show regionally specific interactions, speaking to the integration between inter- 


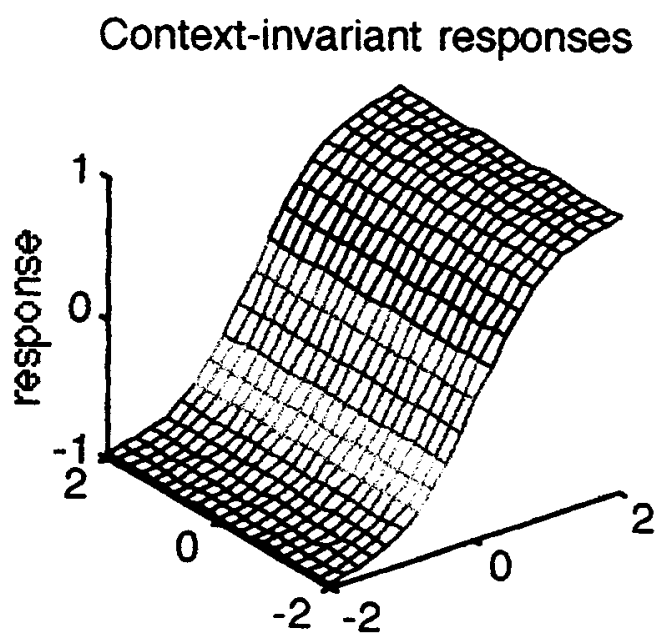

Context-sensitive responses

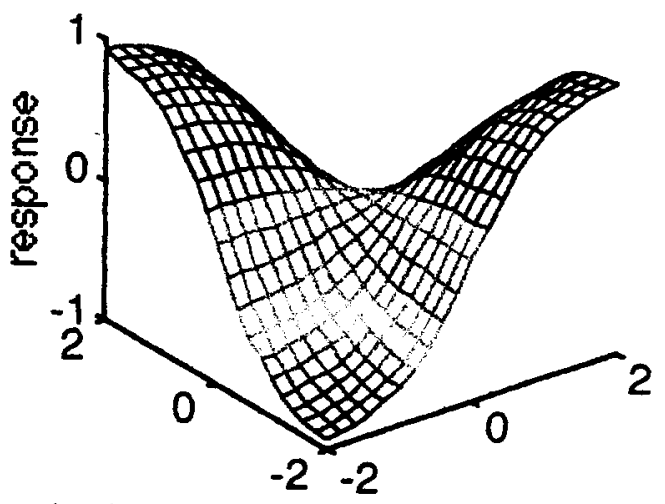

context

Figure 1 (Friston). Schematic to illustrate the relationship between interactions and context-sensitive responses. The upper panel shows the response of a neuronal system to changes in some attribute to which it is sensitive. In this instance the responses are insensitive to the context (i.e., there are no interactions between attribute and context. Conversely the responses in the lower panel evidence strong interactions and context-sensitivity. These response profiles can be thought of as "receptive fields."

pretive sentence processing and the mnemonic processes engaged by digit recall. Finding such effects would not detract from inferences about segregation in other brain areas but would speak to the integration, or coordination, of segregated processing systems (Friston et al. 1996). Although, to my knowledge this experiment has never been carried out, it seems extremely well justified by the psychological background provided in Caplan \& Waters.

\section{Interpretative and post-interpretative processes in sentence comprehension}

\section{Edward Gibson ${ }^{a}$ and Rose Roberts ${ }^{b}$}

aDepartment of Brain and Cognitive Sciences and Department of Linguistics and Philosophy, Massachusetts Institute of Technology, Cambridge, MA 02139 and; ${ }^{\mathrm{b}}$ Department of Brain and Cognitive Sciences, Massachusetts Institute of Technology, Cambridge, MA 02139. gibson@psyche.mit.edu rose@psyche.mit.edu
Abstract: We discuss several issues raised by Caplan \& Waters's distinction between interpretative and post-interpretative processes in sentence comprehension, including the nature and properties of the two systems, problems with measuring their respective capacities, and the relationship between the hypothesized separate-language-interpretation-resource (SLIR) and the general verbal working memory system that supports postinterpretive processing.

\section{Text removed due to third party copyright}

